\title{
Design and Control of In-Vivo Magnetic Microrobots
}

\author{
K. Berk Yesin, Philipp Exner, Karl Vollmers, and Bradley J. Nelson \\ Institute of Robotics and Intelligent Systems, \\ Swiss Federal Institute of Technology (ETH) Zurich, Switzerland \\ \{byesin, kvollmers, bnelson\}@ethz.ch, exnerp@student.ethz.ch
}

\begin{abstract}
This paper investigates fundamental design, modeling and control issues related to untethered biomedical microrobots guided inside the human body through external magnetic fields. Immediate application areas for these microrobots include cardiovascular, intraocular and inner-ear diagnosis and surgery. A prototype microrobot and steering system are introduced. Experimental results on fluid drag and magnetization properties of the robots are presented along with an analysis of required magnetic fields for application inside blood vessels and vitreous humor.
\end{abstract}

\section{Introduction}

The state-of-the-art in MEMS technology is progressing from individual, chiplevel microsensor and microactuator devices to complete integrated microrobot systems. These types of systems will impact future minimally invasive surgical techniques by providing sub-mm untethered biomedical microrobots capable of performing a number of new procedures. The benefit will be even less injury to the patient resulting in correspondingly faster recovery times. Proven MEMS technologies such as micro-needles, micro-pumps and force and chemical sensors will be carried on-board for a variety of surgical and diagnostic tasks. Possible areas of application for these microrobots include cardiovascular, intraocular and inner-ear diagnosis and surgery.

Although electronic and mechanical systems have been miniaturized by VLSI and MEMS technologies, no counterpart to these exists for electro-chemical energy storage. Currently, the only viable option for the actuation and steering of such a microrobot is external energy transfer. The use of magnetic fields generated ex-vivo for energy transfer and actuation can provide a solution to this problem. In this paper a prototype microrobot system that was designed to investigate this idea is introduced. The nature of magnetic and viscous drag forces are discussed and experimental results on the magnetic and drag properties of the microrobots are presented. With these results, the necessary magnetic field strengths for application inside different body-fluids are analyzed. Finally, a strategy for magnetic steering is proposed and a prototype steering system is presented. 


\section{Microrobot with Hybrid MEMS Design}

The microrobot prototype is a three dimensional structure built by microassembly of individual parts shown in Figure 1(a). Currently four different types of robots are being assembled from a combination of these parts and bonded with UV activated glue (Figure 1(c)]. The "winged-ellipsoid" shape has an axis of symmetry along the long axis of the ellipsoid. An external magnetic field acts to align and pull the robot along this axis (i.e. magnetic torque and force) due to the shape anisotropy effect, much like a needle always becoming magnetized along its long axis. On the other hand, the winged shape acts to reduce the side-ways drift of the microrobot by increasing the fluid drag along the axes perpendicular to the long axis.

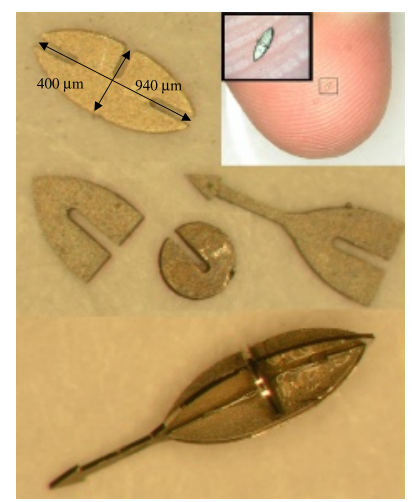

(a)

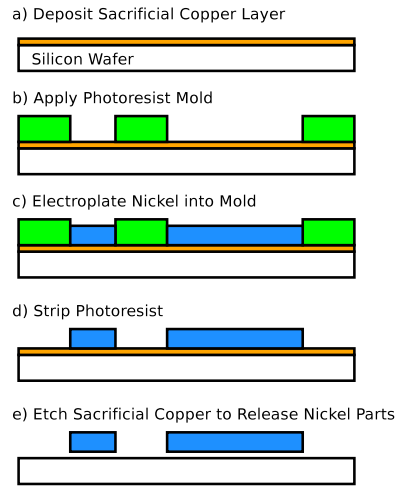

(b)

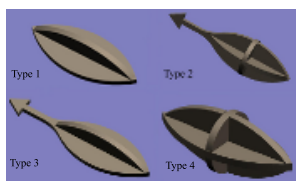

(c)

Fig. 1. a) Nickel microrobot components and an assembled microrobot. The inset shows another microrobot on a finger tip. b) Manufacturing steps for the microrobots. c) Different configurations of microrobots.

The significant advantage of the hybrid design is that the individual parts of the assembly can be produced with standard MEMS manufacturing processes which create planar geometries. This way, different sub-systems of the robot can be manufactured using the most suitable process for the purpose. Figure 1(b) shows the steps of the micromanufacturing process for the nickel parts. Initially the wafers are cleaned and metalized with a titanium adhesion layer and sacrificial copper seed for subsequent electroplating. The thickness of the seed layer is $300 \mathrm{~nm}$. After metallization the wafer is covered with a $80 \mu \mathrm{m}$ thick layer of negative photoresist that is patterned to form the microrobot parts. The nickel parts are then deposited inside a bench top plating system at the rate of $1 \mu \mathrm{m} / \mathrm{min}$ to a thickness of $50 \mu \mathrm{m}$. Following the plating step, the photoresist is stripped and the copper seed layer is etched in a solution of ammonium persulfate which does not attack nickel at low concentrations. 


\section{Wireless Actuation Through Ex-Vivo Magnetic Fields}

\subsection{Nature of Magnetic Forces}

The primary vectors that define the magnetostatic field in magnetized matter are (external) magnetic field strength, $\boldsymbol{H}(\mathrm{A} / \mathrm{m})$, magnetization of the matter $\boldsymbol{M}(\mathrm{A} / \mathrm{m})$ and magnetic flux density $\boldsymbol{B}$ (Tesla). The relationship between these vectors is

$$
\boldsymbol{B}=\mu_{0}(\boldsymbol{H}+\boldsymbol{M})
$$

where $\mu_{0}$ is the magnetic permeability of free space defined as $4 \pi \times 10^{-7} \mathrm{Tm} / \mathrm{A}$. For the idealized case of linear, isotropic and homogeneous media the following relationships simplify (11) as

$$
\begin{gathered}
\boldsymbol{M}=\chi \boldsymbol{H} \\
\boldsymbol{B}=\mu_{0}(1+\chi) \boldsymbol{H}=\mu_{0} \mu_{r} \boldsymbol{H}
\end{gathered}
$$

where $\chi$ and $\mu_{r}$ are the susceptibility and relative permeability of the media, respectively. In general, these values are not constant but change with magnetization, approaching zero as the magnetization reaches a material dependent limit called the saturation magnetization $M_{s}$. Within the saturation limits, the permeability can be thought as an amplification factor that creates a net magnetic field inside the matter through an external field.

The magnetic force and torque that are exerted on an object with uniform magnetization $\boldsymbol{M}$ in a magnetic field with flux density $\boldsymbol{B}$ are defined as

$$
\begin{gathered}
\boldsymbol{F}_{\boldsymbol{m}}=V_{m}(\boldsymbol{M} \cdot \nabla) \boldsymbol{B} \\
\boldsymbol{\tau}_{\boldsymbol{m}}=V_{m} \boldsymbol{M} \times \boldsymbol{B}
\end{gathered}
$$

where $V_{m}$ is the volume of the magnetized object. Notice that the magnetic torque is dependent on $\boldsymbol{B}$ whereas the magnetic force is dependent on the gradient of $\boldsymbol{B}$. Equations (4) and (5) indicate that magnetic forces are volumetric. Therefore, the required fields and field gradients to exert a certain torque and force on a magnetized object increase rapidly as the object gets smaller.

\subsection{Magnetic Properties of the Microrobot}

The relationship between the external magnetic field and the resulting magnetization of the robot is dependent on geometry and material properties. However, the magnetic properties of the material are also significantly affected by the manufacturing process, which is difficult to model. Therefore, it was necessary to perform experimental evaluation of the magnetization curve for the microrobots in order to obtain more reliable values. The microrobots were tested using a coercivity spectrometer device. The results of these tests covered the full range of magnetization as shown in Figure 2. The robots reached a saturation magnetization of 5 to $8 \times 10^{5} \mathrm{~A} / \mathrm{m}$ with a $0.2 \mathrm{~T}$ external field. 

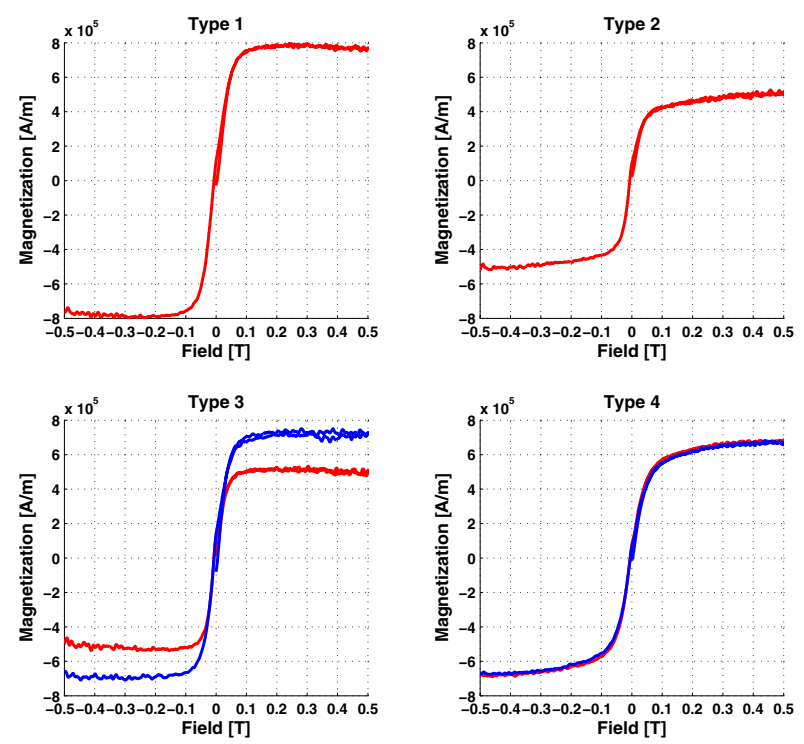

Fig. 2. Magnetization curves for the different types of microrobots (Figure 1(c)). Types 3 and 4 were tested with two different robots.

\subsection{Fluid Drag Forces on the Microrobot}

Other than the magnetic forces analyzed above, gravitation/bouyancy and viscous fluid drag forces are also acting on the microrobot. The drag force on a spherical object in laminar flow regime (i.e. $R e<1.0$ ) can be expressed as

$$
F_{d}=3 \pi D \mu_{f} \nu
$$

where $D$ is the diameter of the sphere, $\mu_{f}$ is the dynamic viscosity of the fluid and $\nu$ is the relative velocity of the robot with respect to the fluid media. Equation (6), also known as Stoke's Drag, is frequently used for calculating the drag force on magnetic beads and other small particles, approximating their shape as a sphere 1. In addition to the drag force, a net buoyancy force will be acting on the robot

$$
F_{b}=V_{r}\left(\rho_{r}-\rho_{f}\right) g
$$

where $V_{r}$ and $\rho_{r}$ are the density and volume of the robot respectively and $g$ is the gravitational acceleration. The most important outcome of (44), (5), (6) and (7) is that, whereas magnetic and buoyancy forces are volumetric, the fluid drag forces are linearly dependent on the size. For this reason, as the size of the robot gets smaller the required field gradient to move at a particular speed rapidly increases.

\subsection{Viscous Drag Force Experiments}

We performed experiments in order to quantify the drag forces on the wingedellipsoid shape of the microrobots. The experiments were conducted by releasing 

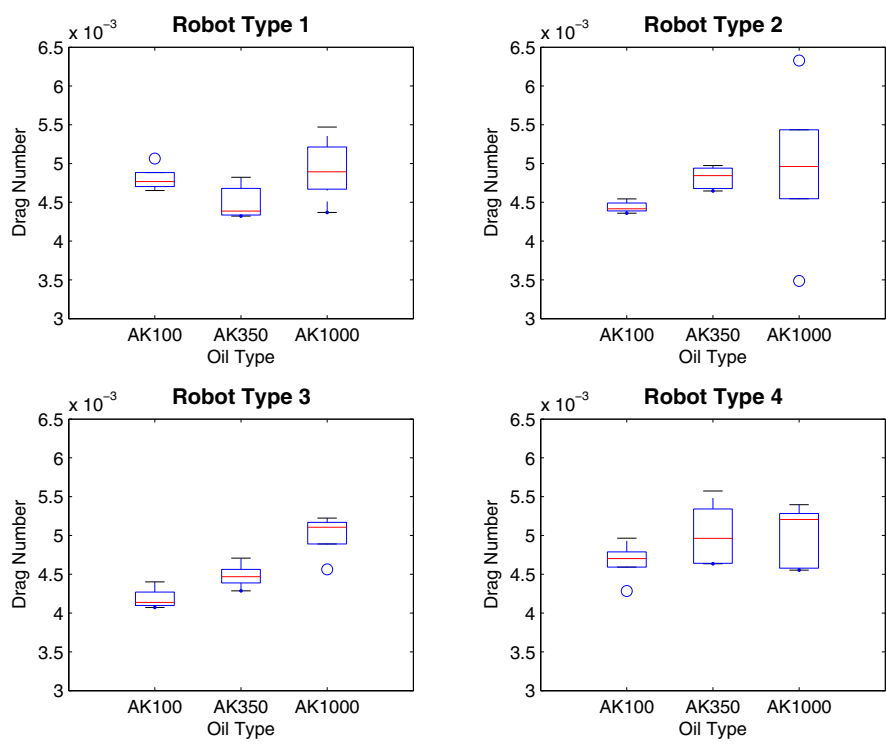

Fig. 3. Drag numbers calculated for each robot type for oils of three different viscosities

the microrobots inside a chamber filled with a fluid of known viscosity and observing them with a microscope-camera in order to determine their terminal velocity under the net effect of fluid drag, gravity and buoyancy forces. The experiments were performed using three different silicon oils AK100, AK350 and AK1000, each having room temperature dynamic viscosities of 100, 350 and $1000 \mathrm{cP}$ (centiPoise), respectively. The actual viscosity values were corrected for the measured temperature of the chamber. The experimental setup was verified using steel ball-bearing spheres of known size and weight. The measurements for the steel spheres were within $5 \%$ of the calculated values.

The results of experiments indicated a linear dependency of the drag force on velocity, similar to the case of a sphere. A drag number, $D_{N}$ was calculated as $D_{N}=F_{d} / \mu_{f} \nu$ for each of the four types of the robot. The resulting data are shown in Figure 3. The boxplots present the distribution of the repeated measurements. The height of the box indicates the interquartile range between $25 \%$ and $75 \%$ percentiles of the data, while the line inside the box shows the median. The drag number measurements show a variation of about $15 \%$, clustered around a value of $4.5 \times 10^{-3} \mathrm{~m}$. If this value is converted to an effective sphere diameter through the relation $D_{N}=3 \pi D$, we find a diameter of $477 \mu \mathrm{m}$ which is close to the cross-section diameter $(400 \mu \mathrm{m})$ of the ellipsoid shape.

\subsection{Required Magnetic Fields in Body Fluids}

With the analysis of magnetic and viscous drag properties of the microrobots, the necessary magnetic field strengths for medical applications can be estimated. For 

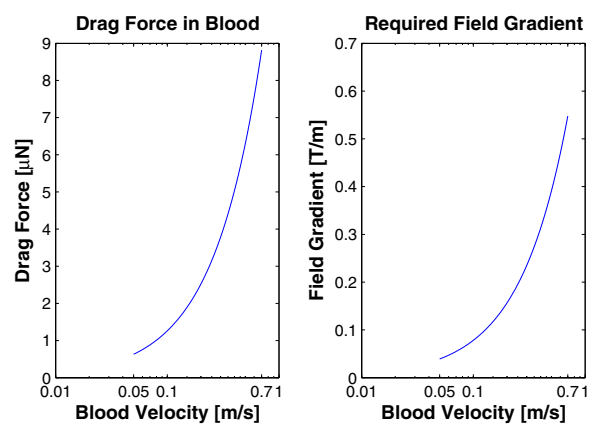

(a)
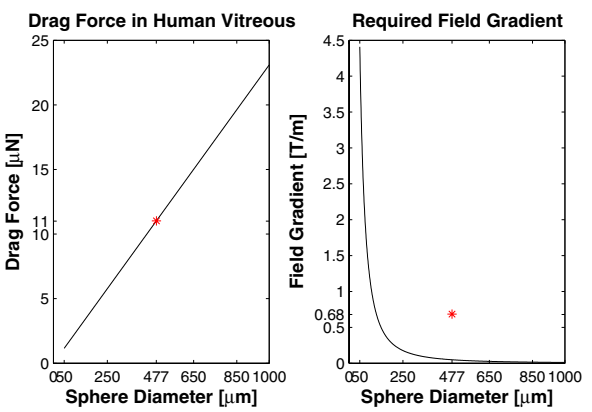

(b)

Fig. 4. a) Drag forces on the microrobot in human blood and the required field gradient to resist the fluid drag. b) Drag forces on a spherical magnet moving at $0.5 \mathrm{~mm} / \mathrm{s}$ in the human vitreous and the required field gradient for various sphere diameters. Magnetization is $\boldsymbol{M}=5 \times 10^{5} \mathrm{~A} / \mathrm{m}$ and viscosity is $4900 \mathrm{cP}$. The values for the prototype microrobot are shown with *.

this analysis the microrobots will be assumed to be magnetized to saturation at $5 \times 10^{5} \mathrm{~A} / \mathrm{m}$. The volume of a Type 3 robot will be considered $\left(3.22 \times 10^{-11} \mathrm{~m}^{3}\right)$. For fluid drag, the average drag number of $4.5 \times 10^{-3} \mathrm{~m}$ is used as an approximation.

For cardiovascular applications, the fluid media that the microrobot moves in is blood. The flow velocity of blood in the human body ranges between 0.05 $\mathrm{m} / \mathrm{s}$ in the capillaries to $0.7 \mathrm{~m} / \mathrm{s}$ at the exit of the aorta with a viscosity of about $2.8 \mathrm{cP}[2]$. Figure 4(a) shows the drag forces on the microrobot and the required field gradient to balance the robot against the flow.

The human eye is another challenging workspace for a biomedical microrobot. The vitreous humor fills the posterior cavity of the eye between the lens and the retina and has the properties of a viscoelastic liquid with high viscosity [3]. A complex model of the vitreous with spring and dashpot elements representing the elastic and viscous characteristics of the material was developed [4] [5. The viscosity of the Maxwell-dashpot element of this model which represents the irreversible flow of the material under constant stress is the main source of the viscous drag forces on the robot. The mean values of viscosity were $1398 \mathrm{cP}$ at the anterior, $2179 \mathrm{cP}$ at the central and $4862 \mathrm{cP}$ at the posterior regions. Figure 4(b) shows the drag forces on a spherical shaped robot with a diameter between 50 to $1000 \mu \mathrm{m}$ and magnetization $5 \times 10^{5} \mathrm{~A} / \mathrm{m}$ moving inside the vitreous at a speed of $0.5 \mathrm{~mm} / \mathrm{sec}$. The viscosity at the posterior region is used. In addition, the necessary field gradient to balance the drag forces is also shown. The location of the prototype microrobot with the winged-ellipsoid shape is also indicated.

\section{Magnetic Steering System}

Equations (44) and (5) suggest that controlled external magnetic fields can be used to induce torques and forces on a magnetized object and control its orien- 


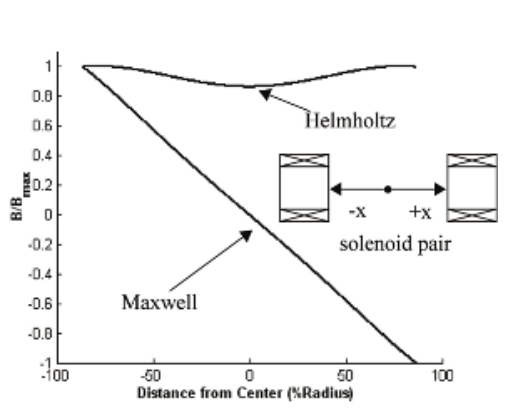

(a)

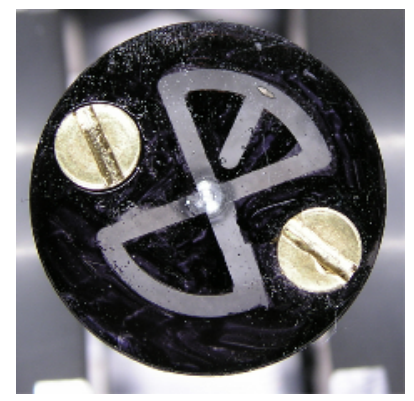

(c)

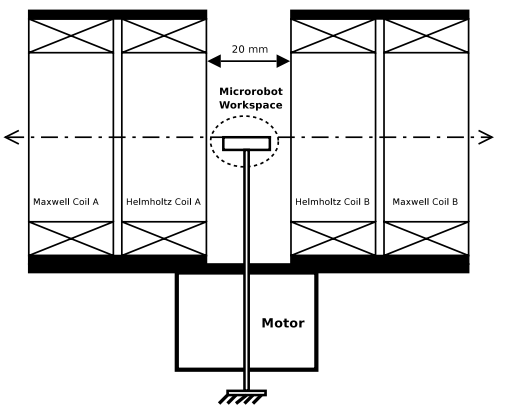

(b)

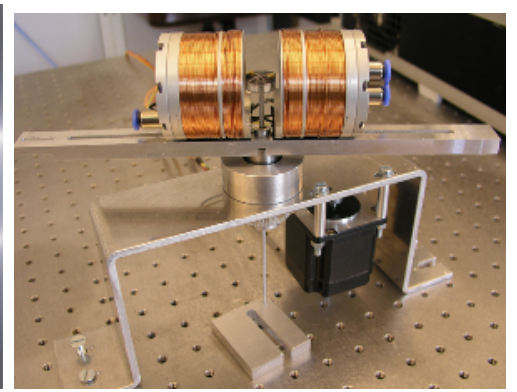

(d)

Fig. 5. a) Normalized magnetic field along the central axes of Helmholtz and Maxwell coils. b)Steering system with co-axial Maxwell and Helmholtz coils. A maze structure is fixed at the center of the workspace between the rotating coils. c) Microrobot steering maze. The channels are $1000 \mu \mathrm{m}$ wide and $300 \mu \mathrm{m}$ deep. The microrobot is also visible at the upper part. d) Magnetic steering system.

tation and position. An important issue related to control is the highly nonlinear nature of the field and gradients that are created by electromagnet coils. One way of reducing the effect of such nonlinearities is to create uniform magnetic fields and field gradients using various coil configurations [6]. For example, the Helmholtz coil configuration consists of two identical coils that are placed on the same axis and separated by a distance equal to the radius of the coils. This arrangement generates a uniform field close to the center of the coil pair when current passes in the same direction in both coils. A variation of this configuration with opposing direction of currents is the Maxwell coil which generates a uniform gradient. Figure 5(a) shows the plot of the fields of Helmholtz and Maxwell coils. Both of these coils are commonly used in MRI systems. Although, the uniform fields of commercial MRI machines are quite high (0.5-2.0 T), the gradient fields they can generate continuously are in the 0.01 to $0.05 \mathrm{~T} / \mathrm{m}$ range.

The magnetic steering principle was investigated using a coil system as illustrated in Figure 5(b) The magnetic torque and force on the microrobot were controlled independently through the Maxwell and Helmholtz fields, respectively. 
This way the orientation and direction of motion of the microrobot could be commanded on a two dimensional plane. Steering tests were performed in a water filled, maze-like structure etched into a plastic substrate (Figure 5(c)). The maze was fixed at the center of the rotating coils, and a microscope camera system was used to obtain a top view (Fig. 5(c). The orientation of the coils were controlled through a PC interface. Numerous trials with the system confirmed that the independent orientation/thrust control principle was successful. The microrobot was observed to follow the orientation of the coils without translating unless the Maxwell coils are energized.

\section{Conclusions}

This paper investigated the concept of steering sub-mm sized untethered microrobots inside body fluids with external magnetic fields. A prototype microrobot was introduced. Estimations on the necessary field gradient to resist fluid drag forces in different body fluids showed that a minimum gradient of about $0.7 \mathrm{~T} / \mathrm{m}$ would be necessary. A magnetic steering principle based on independent control of orientation and thrust with constant field and field-gradient generating coils was successfully tested. These results confirm that magnetic actuation is a suitable mode of energy transfer for untethered biomedical microrobots.

\section{References}

1. D.L. Holligan, G.T. Gilles, J.P. Dailey, "Magnetic Guidance of Ferrofluidic Nanoparticles in an In Vitro Model of Intraocular Retinal Repair", IOP Nanotechnology, vol. 14, pp. 661-666, 2003.

2. A. Despopoulos, S. Silbernagl, Color Atlas of Physiology, New York: Thieme Medical Publishers, pp. 156, 1991.

3. T.V. Chirilla, Y. Young, "The Vitreous Humor", Handbook of Biomaterial Properties", J. Black and G. Hastings et al., Chapman\& Hall, London, 1998.

4. B. Lee, M. Litt, G. Buchsbaum, "Rheology of the Vitreous Body. Part I: Viscoelasticity of Human Vitreous", Biorheology, vol. 29, pp. 521-533, 1992.

5. B. Lee, Comparative Rheological Studies of the Vitreous Body of the Eye, Ph.D. Thesis, University of Pennsylvania, 1992.

6. J. Jin, Electromagnetic Analysis and Design in Magnetic Resonance Imaging, Florida: CRC Press, 1999. 